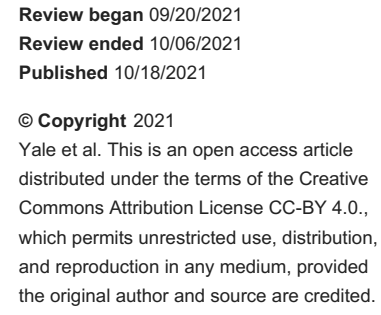

\section{How to Classify, Teach, and Learn Ophthalmic Eponyms}

\author{
Steven Yale ${ }^{1}$, Halil Tekiner ${ }^{2}$, Eileen S. Yale ${ }^{3}$ \\ 1. Internal Medicine, University of Central Florida College of Medicine, Orlando, USA 2. Department of the History of \\ Medicine and Ethics, Erciyes University School of Medicine, Melikigazi, TUR 3. General Internal Medicine, University \\ of Florida College of Medicine, Gainesville, USA
}

Corresponding author: Steven Yale, steven.yale.md@gmail.com

\section{Abstract}

\section{Introduction}

There are limited educational studies on effective ways to teach and learn medical eponyms. While there is no consensus on how to best address this issue, developing novel strategies to teach medical eponyms has become critical in many branches of medicine, including ophthalmology.

\section{Materials \& Methods}

An ophthalmologic eponymic database was created using eight source texts (e.g., books, encyclopedias, and dictionaries) and included the year the eponym was introduced, related name, nationality, specialty, and the eponym's description. PubMed database with a Medical Subject Headings (MeSH) keyword for "eponym" and "eye" and "ophthalmology" and a Google search for a combination of related keywords was also performed. A careful biographical search was conducted for each name in the second phase to obtain further biographical details. Inclusion criteria for eponyms in the dataset were: i) named after at least one person, ii) identified as a specific medical term in the literature, iii) related to any field of medicine. Names derived from art, history, mythology, patient, family, chemistry, botany (or other fields outside of medicine) were excluded. The three authors independently screened to eliminate duplicated names and ensure eligible names met inclusion and exclusion criteria.

\section{Results}

A total of 1,257 unique ophthalmologic eponyms representing $8.8 \%$ of 14,332 medical eponyms were identified. Three-hundred fifty-one of 743 (47.2\%) eponyms were named after ophthalmologists representing 36 countries. The United States of America and Germany comprised the largest fraction of nationalities (40.2\%), not necessarily representing their birthplace. Signs, syndromes, and diseases composed the largest category (45.8\%) of eponymous ophthalmologic names.

\section{Discussion}

The current volume of eponymous names impedes the ability of a learner to retain this information. Classifying eponyms based on form, intention, or function, provides a more refined method for placing eponyms in their respective categories. Teaching eponyms by enumerating their historical content, demonstrating the correct performance of the eponym, assessing the technique, and providing feedback, affords the learner a more fruitful and meaningful learning experience. Understanding the context of the signs, syndrome, or techniques further allows the learner to gain insights into the clinical application of eponyms in diagnostic decision-making.

\section{Conclusion}

The teaching model proposed incorporates key aspects that may facilitate retention and recall of the eponymous name. The model includes imparting historical knowledge about the person who described the sign, technique, or process; demonstrating the correct procedure as originally reported; and coaching to ensure that the appropriate skill is mastered. Before abandoning eponyms, it is first necessary to understand their efficacy, effectiveness, usefulness, and role in clinical medicine.

Categories: Internal Medicine, Medical Education, Ophthalmology

Keywords: student education, syndromes, physical signs, eponyms, ophthalmology

\section{Introduction}

Eponyms provide a rich cultural perspective to the literature and remind us about our historical medical heritage. Despite this, the routine use of eponyms remains controversial, with not all physicians embracing their widespread use [1-3]. Unfortunately, ophthalmologic eponyms have not been well studied, and there is a paucity of information available for only a few eponyms in clinical medicine $[4,5]$. Before abandoning 
eponyms, it is incumbent that we further study them in order to determine their reliability, validity, and applicability in clinical practice. Eponyms found to be valid should be further classified, based on expert advice within the various subspecialties, to facilitate teaching and learning. This paper aims to formulate and propose a teaching model that provides a meaningful way for classifying and teaching ophthalmological eponyms. We recognize gaps in our knowledge about how to teach eponyms as there are limited studies available. Furthermore, the principles applied to the ophthalmologic literature are also applicable to other specialties of medicine.

\section{Materials And Methods}

Data were obtained from research utilizing primarily eight sources representing medical or eponymic books, dictionaries, encyclopedias, indexes, medical periodicals, and one online source. The following criteria were considered in selecting eponyms: i) the eponym should be named after at least one person, ii) the eponym should be identified as a specific medical term in the literature, and iii) the eponyms should be related to any field of medicine. Names derived from art, history, mythology, patient or family names were excluded from the list identified. Moreover, eponymic names related to chemistry, biology, botany (or other fields outside of medicine) were also excluded.

In the first phase, an eponymic database was created. The eight sources were scanned by one of the authors (Halil Tekiner), identifying names, year introduced, related name, nationality and specialty, and the eponym description. The PubMed database was searched with a Medical Subject Headings (MeSH) keyword for "eponym" and "eye" and "ophthalmology" in humans published from inception to June 1, 2021. A Google search for a combination of related keywords was also performed to find any other names not appearing in text sources. A biographical search was conducted for each name in the second phase to obtain further biographical details. Obituaries were extremely helpful in this regard. For those names whose gender was not apparent, a photographic search was also conducted. The three authors independently screened to eliminate duplicated names and ensure eligible names met inclusion and exclusion criteria.

This type of research has its limitations. Despite our efforts, it is inevitable to have several names missed in this list or the derivation undetermined, especially those known only with their surname or the initial letters of their names. Therefore we cannot claim this list is precisely complete in its current form.

\section{Results}

We identified and classified 1,257 (8.8\%) unique ophthalmologic eponyms representing 14,332 total eponyms in the eponymous literature [6-13]. We created eight categories within this classification schema as this would limit the number of categories making it easier to manage and learn. Each eponym was assigned to a single category to avoid overlap, even though some fit into more than one. For example, Westphal pupillary reflex is both a clinical and physiologic phenomenon but was assigned to the clinical one, as it is more relevant to a practicing physician. Categories were arranged based on common themes. The category algorithm, classification, formula, theory, and law all involve problem-solving techniques. Clinical symptoms are objective or subjective findings or phenomena. Pathologic findings were grouped with clinical symptoms as they represent the histopathologic component to an objective or subjective condition. The words device, instrument, product, and supply are in reference to a larger category named tools, as a device may be an instrument or equipment, and an instrument is a device. With this exception, all the remaining eponyms received one qualifier within a category.

Signs, syndromes, and diseases represented the largest category at 576 (45.8\%), followed by those including devices, instruments, medical products and supplies at 260 (20.7\%), clinical/pathological aspects of disease at 185 (14.7\%), techniques at 127 (10.1\%), and other subcategories (Table 1). 


\section{Cureus}

\section{Classification}

Algorithm, classification, formula, theory, law

Anatomic or physiologic

Clinical symptoms or pathologic findings

Device, instrument, product, supply (tool)

Infectious agents

Sign, syndrome, or disease

Technique

Test or treatment
Frequency of unique eponyms

6

57

185

260

11

576

127

35

TABLE 1: Classification of ophthalmologic eponyms

Named after 743 professionals (including 351 ophthalmologists) from 36 countries, the vast majority (97.9\%) of these are names honored men, reflecting the preponderance of men in this specialty before the midtwentieth century. Interestingly, the nationality of these individuals, not necessarily the country from which the eponym was written, were American (20.9\%), German (19.3\%), and Austrian (10.2\%); followed by French (9.8\%), Swiss (7.6\%), and English (7.1\%) among many others.

\section{Discussion}

Eponyms used in the context of clinical medicine are honorific terms bestowed to an individual(s) who identified or discovered a disease, sign, symptom, syndrome, test, or finding. They may also represent a designation of an anatomical structure, devices, procedures or techniques, views or phenomena, treatments, classifications or indexes, prediction rules, laws or principles, or algorithms. Eponymic signs and findings are rarely pathognomonic by themselves. When used in combination with other symptoms, signs, and physical findings, it assists in diagnosis. Their use should be strictly reserved to honor individuals whose contribution(s) embody the rich tradition of the art of medicine for centuries.

There are drawbacks to the use of medical eponyms, which have led to reservations regarding their use. Limitations with eponyms include inaccessibility of the original publication or presentation because it was written in a different language or presented at a conference or in a monograph, historical misinformation, lack of attribution of all authors, naming (e.g., misspelled, a middle name used, multiple renditions of surname, and the same name which may have different meanings), and transcription challenges from nonLatin scripts such as Arabic, Greek, and Russian or sometimes from umlaut letters (ä, ö, ü) in the extended Latin alphabet. Other issues regarding eponyms are the routine use by authors of the possessive form of the name and inclusion within the literature of eponymic names derived from individuals who committed atrocities against humanity or bore racist or antisemitic remarks. The latter has improved as of more recent times as there has been increased awareness and confirmation of these claims. As a result, diseases were renamed and replaced with more descriptive terms (e.g., Wenger granulomatosis to granulomatous with polyangiitis or Reiter syndrome to reactive arthritis). The possessive form of the eponymous name continues to be used in the literature even though its use should be restricted to cases whereby the person had the sign, disease, or syndrome for which they described. The most highly recognized example of where the possessive form is an acceptable designation is Trousseau's sign named for Armand Trousseau (1801-1867), who developed phlebitis occurring in association with a gastric tumor [14].

It is unrealistic to think that hundreds of ophthalmologic eponymic names could be taught, processed, remembered, and recalled. This is compounded in that many eponymous names having more than one designation in the same category. Furthermore, it may not be recognized that an eponym may also be named for both a father and son, as in Sturge-Weber syndrome or von Hippel-Lindau disease. Several papers recommend standardizing terminology when naming new and existing morphological abnormalities and new diseases that can also be applied when classifying ophthalmologic eponyms [15-17]. This serves as the basis for assisting in identifying the framework for naming eponyms. The critical designations to consider are that eponyms be limited to one proper name and no more than three names with the last name(s) used, and authorship preferably limited to include those in the first three positions of the manuscript or monographs. Initials and acronyms [(e.g., acute retinal necrosis (ARN), congenital hypertrophy of the retinal pigment epithelium (CHRPE), and acute posterior multifocal placoid pigment epitheliopathy (APMPPE)] should be avoided. Eponyms that are currently obsolete (e.g., Donder glaucoma, Donder ring, Doyne honeycomb choroidopathy, Filatov operation) should be excluded from ophthalmologic terminology. 


\section{Cureus}

Despite these limitations, which apropos constitute a small portion of the eponymous corpus, we believe that eponyms should be retained and further studied unless there is compelling evidence to the contrary. We concur with the words of Sir Gordon Gordon-Taylor (1878-1960), a pioneering British surgeon and past President of the Royal Society of Medicine, “This may sound antiquated, but it goes against me to sacrifice names which for centuries have proved to be good and useful. The honorable names of our science are thereby fixed in the memory of posterity, and through them, there is awakened in the student a certain historical interest which stimulates him to further investigation" [18].

The field of ophthalmology is replete with eponyms that have been assigned to nearly every conceivable structure within the eye or as part of a systemic process that secondarily involves the eye. Interest in eponyms is likely to grow in recent years, with many journals publishing articles on ophthalmologic eponyms such as Eales disease and Sjögren syndrome or eponyms named to honor female ophthalmologists [19-22].

Clarifications regarding eponyms and their clinical applications can only come about through further studies that examine their validity, reliability, and reproducibility. Physicians make cognitive errors in clinical decision-making that involve biases and heuristic shortcuts. This may be further perpetuated based on anchoring and premature closure, which leads physicians astray from identifying the correct diagnosis. Other errors are linguistic, based on a physician's lack of knowledge regarding the meaning of a structure, process, disease, symptom, syndrome, or sign. These failures include physician's inability to distinguish Bell (Charles Bell, 1774-1842) palsy as idiopathic facial nerve paralysis from other known causes of peripheral facial nerve paralysis, or that William John Adie (1886-1935) described the findings of areflexia found predominantly in women with a known tonic pupil and thus constitutes a syndrome (Adie syndrome), a constellation of signs, symptoms, and findings, "All the evidence seems to me to support the notion that the tonic convergence reaction in pupils apparently inactive to light is a thing apart. The peculiar extra-ocular phenomena (symptomless areflexia) that are frequently associated with it also suggests that we are confronted by a unique condition" [22]. Therefore, it is incumbent that educators teach these principles to learners to avoid perpetuating the same errors. The question is, what the best method for teaching eponyms is?

Disease, syndromes, and signs will continue to become better defined as they are studied, leading to, in some cases, a more refined and specific disease classification. Eponymically named signs and syndromes by themselves lack sufficient specificity alone for diagnostic purposes. In most cases, they represent only the anatomic (e.g., Leber venous plexus), physiologic (Donder law), or pathologic (e.g., Fuch adenoma) expression of the disease or algorithm (e.g., Helvacioglu reproducibility index), classification (e.g., Mann), theory (e.g., Elschnig), or formula (e.g., Reuss). These eponymic signs, syndromes, findings, devices, and techniques, when used in some cases in combination with other more sophisticated diagnostic tests, assist in better understanding, sorting out, and sorting through, the various disease processes.

Classifying ophthalmologic eponyms based on a mechanistic approach assists the reader to understand better their representation (e.g., anatomic, pathologic, physiologic) and purpose (e.g., test, technique, algorithm, classification, instrument, or device, or operative technique), as shown in Table 2 .

\begin{tabular}{|c|c|c|}
\hline Name & Eponym & Classification \\
\hline Adie, William John (1886-1935) & Adie pupil & clinical symptoms \\
\hline Adie, William John (1886-1935) & Adie syndrome & syndrome \\
\hline Adie, William John (1886-1935) & Adie-Critchley syndrome & syndrome \\
\hline Amsler, Marc (1891-1968) & Amsler chart & tool \\
\hline Amsler, Marc (1891-1968) & Amsler chart marker & tool \\
\hline Amsler, Marc (1891-1968) & Amsler corneal graft operation & technique \\
\hline Amsler, Marc (1891-1968) & Amsler grid & tool \\
\hline Amsler, Marc (1891-1968) & Amsler needle & tool \\
\hline Amsler, Marc (1891-1968) & Amsler scleral marker & tool \\
\hline Amsler, Marc (1891-1968) & Amsler test & test \\
\hline Amsler, Marc (1891-1968) & Amsler-Verry sign & sign \\
\hline Arlt, Carl Ferdinand Ritter von (1812-1887) & Arlt eyelid repair & technique \\
\hline Arlt, Carl Ferdinand Ritter von (1812-1887) & Arlt fenestrated lens scoop & tool \\
\hline
\end{tabular}




\section{Cureus}

Arlt, Carl Ferdinand Ritter von (1812-1887)

Arlt, Carl Ferdinand Ritter von (1812-1887)

Arlt, Carl Ferdinand Ritter von (1812-1887)

Arlt, Carl Ferdinand Ritter von (1812-1887)

Arlt, Carl Ferdinand Ritter von (1812-1887)

Arlt, Carl Ferdinand Ritter von (1812-1887)

Arlt, Carl Ferdinand Ritter von (1812-1887)

Arlt, Carl Ferdinand Ritter von (1812-1887)

Arlt, Carl Ferdinand Ritter von (1812-1887)

Arlt, Carl Ferdinand Ritter von (1812-1887)

Axenfeld, Karl Theodor Paul Polykarpus (1867-1930)

Axenfeld, Karl Theodor Paul Polykarpus (1867-1930)

Behçet, Hulusi (1889-1948)

Behçet, Hulusi (1889-1948)

Behr, Carl Julius Peter (1874-1943)

Behr, Carl Julius Peter (1874-1943)

Behr, Carl Julius Peter (1874-1943)

Behr, Carl Julius Peter (1874-1943)

Bell, Sir Charles (1774-1842)

Bell, Sir Charles (1774-1842)

Bell, Sir Charles (1774-1842)

Bell, Sir Charles (1774-1842)

Bell, Sir Charles (1774-1842)

Best, Franz (1878-1920)

Best, Franz (1878-1920)

Best, Franz (1878-1920)

Bielschowsky, Alfred (1871-1940)

Bielschowsky, Alfred (1871-1940)

Bielschowsky, Alfred (1871-1940)

Bielschowsky, Alfred (1871-1940)

Bielschowsky, Alfred (1871-1940)

Bielschowsky, Alfred (1871-1940)

Bielschowsky, Alfred (1871-1940)

Bielschowsky, Alfred (1871-1940)

Bielschowsky, Alfred (1871-1940)

Bjerrum, Jannik Petersen (1851-1926)

Bjerrum, Jannik Petersen (1851-1926)

Bjerrum, Jannik Petersen (1851-1926)

Bjerrum, Jannik Petersen (1851-1926)

Bowman, Sir William (1816-1892)
Arlt lens loupe tool

Arlt line

pathologic findings

Arlt operation

technique

Arlt pterygium excision

technique

Arlt recess

anatomic

Arlt sinus

Arlt sutures

anatomic

tool

Arlt syndrome

syndrome

Arlt trachoma

pathologic findings

Arlt triangle

Axenfeld calcareous degeneration

pathologic findings

pathologic findings

Axenfeld syndrome

syndrome

Behçet disease

disease

Behçet syndrome

Behr abduction phenomenon

syndrome

clinical symptoms

Behr disease

disease

Behr sign

sign

Behr syndrome

Bell law

Bell nerve

Bell palsy

Bell paralysis

Bell phenomena

Best carmine stain

tes

Best disease

disease

Best macular degeneration

pathologic findings

Bielschowsky disease

Bielschowsky head tilt test

disease

test

Bielschowsky method

technique

Bielschowsky phenomenon

clinical symptoms

Bielschowsky sign

sign

Bielschowsky squint

clinical symptoms

Bielschowsky stain

test

Bielschowsky syndrome

syndrome

Bielschowsky-Dollinger syndrome

syndrome

Bjerrum scotoma

pathologic findings

Bjerrum scotometer

tool

Bjerrum screen

tool

Bjerrum sign

sign

Bowman eye knife 


\section{Cureus}

Bowman, Sir William (1816-1892)

Bowman, Sir William (1816-1892)

Bowman, Sir William (1816-1892)

Bowman, Sir William (1816-1892)

Bowman, Sir William (1816-1892)

Bowman, Sir William (1816-1892)

Bowman, Sir William (1816-1892)

Bowman, Sir William (1816-1892)

Bruch, Karl Wilhelm Ludwig (1819-1884)

Bruch, Karl Wilhelm Ludwig (1819-1884)

Cloquet, Jules Germain (1790-1883)

Cloquet, Jules Germain (1790-1883)

Cloquet, Jules Germain (1790-1883)

Coats, George (1876-1915)

Coats, George (1876-1915)

Coats, George (1876-1915)

Cogan, David Glendenning (1908-1993)

Cogan, David Glendenning (1908-1993)

Cogan, David Glendenning (1908-1993)

Cogan, David Glendenning (1908-1993)

Dalrymple, John (1803-1852)

Dalrymple, John (1803-1852)

Doyne, Robert Walter (1857-1916)

Doyne, Robert Walter (1857-1916)

Doyne, Robert Walter (1857-1916)

Doyne, Robert Walter (1857-1916)

Duane, Alexander (1858-1926)

Duane, Alexander (1858-1926)

Duane, Alexander (1858-1926)

Duane, Alexander (1858-1926)

Eales, Henry (1852-1913)

Edinger, Ludwig (1855-1918)

Elschnig, Anton (1863-1939)

Elschnig, Anton (1863-1939)

Elschnig, Anton (1863-1939)

Elschnig, Anton (1863-1939)

Elschnig, Anton (1863-1939)

Elschnig, Anton (1863-1939)

Elschnig, Anton (1863-1939)

\begin{tabular}{|c|c|}
\hline Bowman iris needle & tool \\
\hline Bowman iris scissors & tool \\
\hline Bowman lacrimal dilator & tool \\
\hline Bowman lacrimal probe & tool \\
\hline Bowman lamellae of cornea & anatomic \\
\hline Bowman membrane & anatomic \\
\hline Bowman probe & tool \\
\hline Bowman strabismus scissors & tool \\
\hline Bruch glands & pathologic findings \\
\hline Bruch membrane & anatomic \\
\hline Cloquet canal & anatomic \\
\hline Cloquet canal remnants & anatomic \\
\hline Cloquet space & anatomic \\
\hline Coats disease & disease \\
\hline Coats ring & pathologic findings \\
\hline Coats syndrome & syndrome \\
\hline Cogan microcystic dystrophy & pathologic findings \\
\hline Cogan sign & sign \\
\hline Cogan syndrome & syndrome \\
\hline Cogan-Reese disease & disease \\
\hline Dalrymple disease & disease \\
\hline Dalrymple sign & sign \\
\hline Doyne choroiditis & pathologic findings \\
\hline Doyne guttate iritis & pathologic findings \\
\hline Doyne honeycomb choroidopathy & pathologic findings \\
\hline Doyne operation & technique \\
\hline Duane parallax test & test \\
\hline Duane retraction syndrome & syndrome \\
\hline Duane syndrome & syndrome \\
\hline Duane test & test \\
\hline Eales disease & disease \\
\hline Edinger-Westphal nucleus & anatomic \\
\hline Elschnig blepharrorrhaphy & technique \\
\hline Elschnig bodies & pathologic findings \\
\hline Elschnig canthorrhaphy & technique \\
\hline Elschnig cataract knife & tool \\
\hline Elschnig conjunctivitis & pathologic findings \\
\hline Elschnig corneal knife & tool \\
\hline Elschnig cyclodialysis spatula & too \\
\hline
\end{tabular}




\section{Cureus}

\begin{tabular}{|c|c|c|}
\hline Elschnig, Anton (1863-1939) & Elschnig dissecting knife & tool \\
\hline Elschnig, Anton (1863-1939) & Elschnig extrusion needle & tool \\
\hline Elschnig, Anton (1863-1939) & Elschnig eye spoon & tool \\
\hline Elschnig, Anton (1863-1939) & Elschnig fixation forceps & tool \\
\hline Elschnig, Anton (1863-1939) & Elschnig iridectomy & technique \\
\hline Elschnig, Anton (1863-1939) & Elschnig lid retractor & tool \\
\hline Elschnig, Anton (1863-1939) & Elschnig pearls & pathologic findings \\
\hline Elschnig, Anton (1863-1939) & Elschnig procedure & technique \\
\hline Elschnig, Anton (1863-1939) & Elschnig pterygium knife & tool \\
\hline Elschnig, Anton (1863-1939) & Elschnig refractor & tool \\
\hline Elschnig, Anton (1863-1939) & Elschnig spots & pathologic findings \\
\hline Elschnig, Anton (1863-1939) & Elschnig syndrome & syndrome \\
\hline Elschnig, Anton (1863-1939) & Elschnig theory & theory \\
\hline Elschnig, Anton (1863-1939) & Elschnig trephine & tool \\
\hline Fleischer, Bruno Otto (1874-1965) & Fleischer corneal ring & pathologic findings \\
\hline Fleischer, Bruno Otto (1874-1965) & Fleischer dystrophy & pathologic findings \\
\hline Fleischer, Bruno Otto (1874-1965) & Fleischer lines & pathologic findings \\
\hline Fleischer, Bruno Otto (1874-1965) & Fleischer ring & pathologic findings \\
\hline Fleischer, Bruno Otto (1874-1965) & Fleischer vortex & pathologic findings \\
\hline Fleischer, Bruno Otto (1874-1965) & Fleischer-Strumpell ring & pathologic findings \\
\hline François, Émile Jules Marie Joseph (1907-1984) & François cloudy central dystrophy & pathologic findings \\
\hline François, Émile Jules Marie Joseph (1907-1984) & François dyscelphalic syndrome & syndrome \\
\hline François, Émile Jules Marie Joseph (1907-1984) & François dystrophy (I) & pathologic findings \\
\hline François, Émile Jules Marie Joseph (1907-1984) & François dystrophy (II) & pathologic findings \\
\hline François, Émile Jules Marie Joseph (1907-1984) & François speckled dystrophy & pathologic findings \\
\hline François, Émile Jules Marie Joseph (1907-1984) & François-Evans syndrome & syndrome \\
\hline Fuchs, Ernst (1851-1930) & Fuchs adenoma & pathologic findings \\
\hline Fuchs, Ernst (1851-1930) & Fuchs atrophy & pathologic findings \\
\hline Fuchs, Ernst (1851-1930) & Fuchs black spots & pathologic findings \\
\hline Fuchs, Ernst (1851-1930) & Fuchs capsule forceps & tool \\
\hline Fuchs, Ernst (1851-1930) & Fuchs capsulotomy forceps & tool \\
\hline Fuchs, Ernst (1851-1930) & Fuchs coloboma & pathologic findings \\
\hline Fuchs, Ernst (1851-1930) & Fuchs corneal dystrophy & pathologic findings \\
\hline Fuchs, Ernst (1851-1930) & Fuchs crypt & anatomic \\
\hline Fuchs, Ernst (1851-1930) & Fuchs dellen & pathologic findings \\
\hline Fuchs, Ernst (1851-1930) & Fuchs dimples & pathologic findings \\
\hline Fuchs, Ernst (1851-1930) & Fuchs disease & disease \\
\hline Fuchs, Ernst (1851-1930) & Fuchs dystrophy & pathologic findings \\
\hline Fuchs, Ernst (1851-1930) & Fuchs epithelial dystrophy & pathologic findings \\
\hline Fuchs, Ernst (1851-1930) & Fuchs grid & tool \\
\hline
\end{tabular}




\section{Cureus}

Fuchs, Ernst (1851-1930)

Fuchs, Ernst (1851-1930)

Fuchs, Ernst (1851-1930)

Fuchs, Ernst (1851-1930)

Fuchs, Ernst (1851-1930)

Fuchs, Ernst (1851-1930)

Fuchs, Ernst (1851-1930)

Fuchs, Ernst (1851-1930)

Fuchs, Ernst (1851-1930)

Fuchs, Ernst (1851-1930)

Fuchs, Ernst (1851-1930)

Fuchs, Ernst (1851-1930)

Goldmann, Hans (1899-1991)**

Goldmann, Hans (1899-1991) **

Goldmann, Hans (1899-1991)**

Goldmann, Hans (1899-1991)**

Goldmann, Hans (1899-1991)**

Goldmann, Hans (1899-1991)**

Goldmann, Hans (1899-1991)**

Goldmann, Hans (1899-1991)**

Goldmann, Hans (1899-1991)**

Goldmann, Hans (1899-1991)**

Gräfe (Graefe), F.W. Ernst Albrecht von (1828-1870)

Gräfe (Graefe), F.W. Ernst Albrecht von (1828-1870)

Gräfe (Graefe), F.W. Ernst Albrecht von (1828-1870)

Gräfe (Graefe), F.W. Ernst Albrecht von (1828-1870)

Gräfe (Graefe), F.W. Ernst Albrecht von (1828-1870)

Graves, Robert James (1796-1853)

Graves, Robert James (1796-1853)

Gunn, Robert Marcus (1850-1909)

Gunn, Robert Marcus (1850-1909)

Gunn, Robert Marcus (1850-1909)

Haab, Otto (1850-1931)

Haab, Otto (1850-1931)

Haab, Otto (1850-1931)

Haab, Otto (1850-1931)

Haab, Otto (1850-1931)

Haab, Otto (1850-1931)

Haab, Otto (1850-1931)

Haab, Otto (1850-1931)
Fuchs heterochromic cyclitis

Fuchs keratome

Fuchs lid

Fuchs phenomenon

Fuchs signs

Fuchs spot

Fuchs stoma

Fuchs superficial marginal keratitis

Fuchs syndrome (I,II)

Fuchs two-way eye syringe

Fuchs uveitis

Fuchs-Kraupa syndrome

Goldmann applanation tonometer

Goldmann contact lens prism

Goldmann expressor

Goldmann goniolens

Goldmann macular contact lens

Goldmann multimirror lens implant

Goldmann perimeter

Goldmann serrated knife

Goldmann three-mirror contact lens

Goldmann-Favre disease

Graefe cautery (electrocautery)

Graefe cystotome

Graefe disease

Graefe knife needle

Graefe sign

Graves ophthalmopathy

Graves orbitopathy

Gunn dots

Gunn pupil

Gunn sign

Haab degeneration

Haab eye knife

Haab line

Haab magnet

Haab needle

Haab reflex

Haab scleral resection knife

Haab senile macular degeneration pathologic findings

tool

pathologic findings

clinical symptoms

sign

pathologic findings

anatomic

pathologic findings

syndrome

tool

pathologic findings

syndrome

tool

tool

tool

tool

tool

tool

tool

tool

tool

disease

tool

tool

disease

tool

sign

clinical symptoms

clinical symptoms

anatomic

clinical symptoms

sign

pathologic findings

tool

pathologic findings

tool

tool

physiologic

tool

pathologic findings 


\section{Cureus}

\begin{tabular}{|c|c|c|}
\hline Haab, Otto (1850-1931) & Haab-Dimmer dystrophy & pathologic findings \\
\hline Harada, Einosuke (1892-1946) & Harada disease & disease \\
\hline Harada, Einosuke (1892-1946) & Harada syndrome & syndrome \\
\hline Henle, Friedrich Gustav Jakob (1809-1885) & Hassle-Henle bodies & pathologic findings \\
\hline Henle, Friedrich Gustav Jakob (1809-1885) & Henle fiber layer & anatomic \\
\hline Henle, Friedrich Gustav Jakob (1809-1885) & Henle glands & anatomic \\
\hline Henle, Friedrich Gustav Jakob (1809-1885) & Henle membrane & anatomic \\
\hline Henle, Friedrich Gustav Jakob (1809-1885) & Henle nervous layer & anatomic \\
\hline Hering, Karl Ewald Konstantin (1834-1918) & Hering afterimage & physiologic \\
\hline Hering, Karl Ewald Konstantin (1834-1918) & Hering test & test \\
\hline Hering, Karl Ewald Konstantin (1834-1918) & Hering theory of color vision & theory \\
\hline Hering, Karl Ewald Konstantin (1834-1918) & Hering-Bielschowsky test & test \\
\hline Hess, Carl von (1863-1923) & Hess capsule iris forceps & tool \\
\hline Hess, Carl von (1863-1923) & Hess chart & test \\
\hline Hess, Carl von (1863-1923) & Hess expressor & tool \\
\hline Hess, Carl von (1863-1923) & Hess eyelid operation & technique \\
\hline Hess, Carl von (1863-1923) & Hess lens scoop & tool \\
\hline Hess, Carl von (1863-1923) & Hess lens spoon & tool \\
\hline Hess, Carl von (1863-1923) & Hess ptosis operation & technique \\
\hline Hess, Carl von (1863-1923) & Hess screen & test \\
\hline Hess, Carl von (1863-1923) & Hess test & test \\
\hline Hippel, Eugen Adolf Arthur von (1867-1939) & Hippel keratoplasty & technique \\
\hline Hippel, Eugen Adolf Arthur von (1867-1939) & Hippel trephine & tool \\
\hline Hippel, Eugen Adolf Arthur von (1867-1939) & Lindau-von Hippel syndrome & syndrome \\
\hline Hippel, Eugen Adolf Arthur von (1867-1939) & von Hippel disease & disease \\
\hline Hippel, Eugen Adolf Arthur von (1867-1939) & von Hippel ulcer & pathologic findigns \\
\hline Horner, Johann Friedrich (1831-1886) & Horner hollow chisel & tool \\
\hline Horner, Johann Friedrich (1831-1886) & Horner pupil & clinical symptoms \\
\hline Horner, Johann Friedrich (1831-1886) & Horner sign & sign \\
\hline Horner, Johann Friedrich (1831-1886) & Horner syndrome & syndrome \\
\hline Horner, Johann Friedrich (1831-1886) & Horner-Trantas dots & pathologic findings \\
\hline Horner, Johann Friedrich (1831-1886) & Horner-Trantas spots & clinical symptoms \\
\hline Hutchinson, Sir Jonathan (1828-1913) & Hutchinson facies & Clinical symptoms \\
\hline Hutchinson, Sir Jonathan (1828-1913) & Hutchinson patch & pathologic findings \\
\hline Hutchinson, Sir Jonathan (1828-1913) & Hutchinson pupil & clinical symptoms \\
\hline Hutchinson, Sir Jonathan (1828-1913) & Hutchinson sign & sign \\
\hline Hutchinson, Sir Jonathan (1828-1913) & Hutchinson syndrome & syndrome \\
\hline Hutchinson, Sir Jonathan (1828-1913) & Hutchinson triad & clinical symptoms \\
\hline Hutchinson, Sir Jonathan (1828-1913) & Hutchinson tumor & pathologic findings \\
\hline
\end{tabular}




\section{Cureus}

Knapp, Herman Jakob (1832-1911)** Knapp, Herman Jakob (1832-1911)* Knapp, Herman Jakob (1832-1911) ** Knapp, Herman Jakob (1832-1911)** Knapp, Herman Jakob (1832-1911)** Knapp, Herman Jakob (1832-1911)** Knapp, Herman Jakob (1832-1911)** Knapp, Herman Jakob (1832-1911) $)^{\star \star}$ Knapp, Herman Jakob (1832-1911)** Knapp, Herman Jakob (1832-1911)** Knapp, Herman Jakob (1832-1911)** Knapp, Herman Jakob (1832-1911)** Knapp, Herman Jakob (1832-1911) Knapp, Herman Jakob (1832-1911)** Koyanagi, Yosizo (1880-1954) Krause, Karl Friedrich Theodor (1797-1868) Laurence, John Zachariah (1829-1870)

Le Fort, Léon Clément (1829-1893)

Leber, Theodor (1840-1917)

Leber, Theodor (1840-1917)

Leber, Theodor (1840-1917)

Leber, Theodor (1840-1917)

Leber, Theodor (1840-1917)

Leber, Theodor (1840-1917)

Leber, Theodor (1840-1917)

Leber, Theodor (1840-1917)

Leber, Theodor (1840-1917)

Leber, Theodor (1840-1917)

Lindau, Arvid Wilhelm (1892-1958)

Maddox, Ernest Edmund (1860-1933)

Maddox, Ernest Edmund (1860-1933)

Maddox, Ernest Edmund (1860-1933)

Maddox, Ernest Edmund (1860-1933)

Marfan, Antoine Bernard-Jean (1858-1942)

Marfan, Antoine Bernard-Jean (1858-1942)

Mikulicz-Radecki, Johannes (Jan) Anton von (1850-1905

Möbius (Moebius), Paul Julius (1853-1907)

Möbius (Moebius), Paul Julius (1853-1907)

Moll, Jacob Antonius (1832-1914)

Moon, Robert Charles (1844-1914)

\begin{tabular}{|c|c|}
\hline Knapp cataract knife & tool \\
\hline Knapp eye speculum & tool \\
\hline Knapp iris hook & tool \\
\hline Knapp iris knife needle & tool \\
\hline Knapp iris repositor & tool \\
\hline Knapp iris scissors & tool \\
\hline Knapp iris spatula & tool \\
\hline Knapp lacrial sac refractor & tool \\
\hline Knapp lens scoop & tool \\
\hline Knapp lid operation & technique \\
\hline Knapp pterygium operation & technique \\
\hline Knapp streaks & pathologic findings \\
\hline Knapp striae & pathologic findings \\
\hline Knapp test & test \\
\hline Vogt-Koyanagi syndrome & syndrome \\
\hline Krause gland & anatomic \\
\hline Laurence-Moon syndrome & syndrome \\
\hline Le Fort fracture & pathologic findings \\
\hline Leber cell & pathologic findings \\
\hline Leber congenital amaurosis & pathologic findings \\
\hline Leber disease & disease \\
\hline Leber hereditary optic atrophy & pathologic findings \\
\hline Leber idiopathic stellate neuroretinitis & pathologic findings \\
\hline Leber miliary aneurysm & pathologic findings \\
\hline Leber plexus & anatomic \\
\hline Leber retinitis & pathologic findings \\
\hline Leber syndrome & syndrome \\
\hline Leber venous plexus & anatomic \\
\hline Lindau disease & disease \\
\hline Maddox prism & tool \\
\hline Maddox rod & tool \\
\hline Maddox rod occluder & tool \\
\hline Maddox rod test & test \\
\hline Marfan syndrome & syndrome \\
\hline Marfan-Madelung syndrome & syndrome \\
\hline Mikulicz syndrome & syndrome \\
\hline Möbius sign & sign \\
\hline Möbius syndrome & syndrome \\
\hline Moll glands & anatomic \\
\hline Laurence-Moon syndrome & syndrome \\
\hline
\end{tabular}




\section{Cureus}

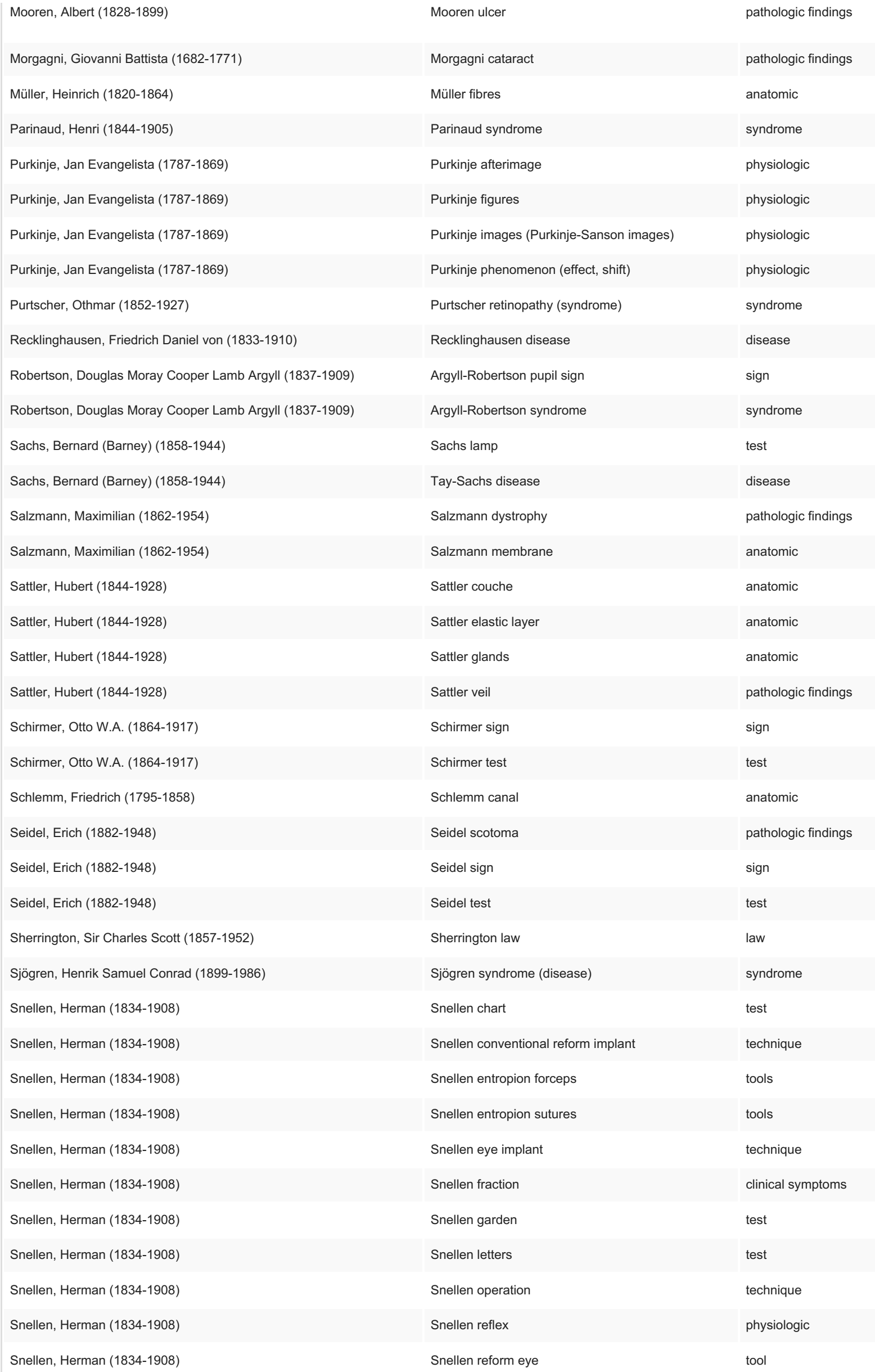




\section{Cureus}

Snellen, Herman (1834-1908)

Snellen, Herman (1834-1908)

Snellen, Herman (1834-1908)

Snellen, Herman (1834-1908)

Snellen, Herman (1834-1908)

Snellen, Herman (1834-1908)

Snellen, Herman (1834-1908)

Sömmerring (Soemmerring), Samuel Thomas von (1755-1830)

Sömmerring (Soemmerring), Samuel Thomas von (1755-1830)

Sömmerring (Soemmerring), Samuel Thomas von (1755-1830)

Sömmerring (Soemmerring), Samuel Thomas von (1755-1830)

Stargardt, Karl Bruno (1875-1927)

Sturge, William Allen (1850-1919)

Sturge, William Allen (1850-1919)

Sturge, William Allen (1850-1919)

Tay, Warren (1843-1927)

Tay, Warren (1843-1927)

Tay, Warren (1843-1927)

Tay, Warren (1843-1927)

Tay, Warren (1843-1927)

Tay, Warren (1843-1927)

Tenon, Jacques-René (1724-1816)

Tenon, Jacques-René (1724-1816)

Terrien, Félix (1872-1940)

Terrien, Félix (1872-1940)

Terrien, Félix (1872-1940)

Terson, Albert (1867-1935)

Terson, Albert (1867-1935)

Terson, Albert (1867-1935)

Terson, Albert (1867-1935)

Treacher Collins, Edward (1862-1932)

Uhthoff, Wilhelm (1853-1927)

Usher, Charles Howard (1865-1942)

Vogt, Alfred (1879-1943)

Vogt, Alfred (1879-1943)

Vogt, Alfred (1879-1943)

Vogt, Alfred (1879-1943)

Waardenburg, Petrus Johannes (1886-1979)

Weber, Frederick Parkes (1863-1962)

Weber, Frederick Parkes (1863-1962)

\begin{tabular}{|c|c|}
\hline Snellen reform implant & tool \\
\hline Snellen sign & sign \\
\hline Snellen soft contact lens & tool \\
\hline Snellen suture & tool \\
\hline Snellen test & test \\
\hline Snellen test types & test \\
\hline Snellen vectis & tool \\
\hline Sömmerring foramen & anatomic \\
\hline Sömmerring ligament & anatomic \\
\hline Sömmerring ring cataract & pathologic findings \\
\hline Sömmerring spot & anatomic \\
\hline Stargardt-Behr disease (syndrome) & disease \\
\hline Sturge-Kalischer-Weber syndrome & syndrome \\
\hline Sturge-Weber disease & disease \\
\hline Sturge-Weber syndrome & syndrome \\
\hline Tay cherry-red spot & pathologic findings \\
\hline Tay choroiditis & pathologic findings \\
\hline Tay sign & sign \\
\hline Tay spot & pathologic findings \\
\hline Tay syndrome & syndrome \\
\hline Tay-Sachs disease & disease \\
\hline Tenon capsule & anatomic \\
\hline Tenon space & anatomic \\
\hline Terrien disease & disease \\
\hline Terrien marginal degeneration & pathologic findings \\
\hline Terrien-Veul syndrome & syndrome \\
\hline Terson forceps & tool \\
\hline Terson glands & anatomic \\
\hline Terson speculum & tool \\
\hline Terson syndrome (disease) & syndrome \\
\hline Treacher Collins syndrome & syndrome \\
\hline Uhthoff phenomenon & clinical symptoms \\
\hline Usher syndrome & syndrome \\
\hline Limbal girdle of Vogt & pathologic findings \\
\hline Vogt anterior mosic crocodile shagreen & pathologic findings \\
\hline Vogt striae & pathologic findings \\
\hline Vogt-Koyanagi-Harada (VKH) syndrome & syndrome \\
\hline Waardenburg-Jonkers syndrome & syndrome \\
\hline Rendu-Osler-Weber syndrome & syndrome \\
\hline Sturge-Kalischer-Weber syndrome & syndrome \\
\hline
\end{tabular}




\section{Cureus}

\begin{tabular}{|c|c|c|}
\hline Westphal, Karl Friedrich Otto (1833-1890) & Strümpell-Westphal disease (pseudosclerosis) & disease \\
\hline Westphal, Karl Friedrich Otto (1833-1890) & Westphal nucleus & anatomic \\
\hline Westphal, Karl Friedrich Otto (1833-1890) & Westphal pupillary reflex & clinical symptoms \\
\hline Westphal, Karl Friedrich Otto (1833-1890) & Westphal-Piltz sign & sign \\
\hline Wintersteiner, Hugo (1865-1918) & Flexner-Wintersteiner rosettes & pathologic findings \\
\hline Zinn, Johann Gottfried (1727-1759) & Zinn artery & anatomic \\
\hline Zinn, Johann Gottfried (1727-1759) & Zinn corona & anatomic \\
\hline Zinn, Johann Gottfried (1727-1759) & Zinn ligament & anatomic \\
\hline Zinn, Johann Gottfried (1727-1759) & Zinn membrane & anatomic \\
\hline Zinn, Johann Gottfried (1727-1759) & Zinn ring & anatomic \\
\hline Zinn, Johann Gottfried (1727-1759) & Zinn vascular circle & anatomic \\
\hline Zinn, Johann Gottfried (1727-1759) & Zinn zone (zonule) & anatomic \\
\hline
\end{tabular}

TABLE 2: Common representative ophthalmologic eponym names

Eponyms that are studied and deemed most relevant to a particular aspect of ophthalmology (e.g., operative, instruments, clinical, and pathologic) should be identified by a panel of experts, emphasized, and taught.

We propose a learning model that involves teaching historical aspects of the person(s) who described the sign, the signs as originally described, and its application, if available, in medical practice. Teaching history imparts purpose to the eponym and, in some cases, a more in-depth understanding of the process or steps involved in identifying the particular finding. Teaching the finding as described by the author avoids misattribution or communication errors. Lastly, the application of the finding in clinical practice should be covered, incorporating known and evolving techniques and technologies to understand the disease or disease process better.

To the best of our knowledge, only one recent study evaluated a method for teaching eponyms. Viveen et al. assessed knowledge involving ten common eponymous questions before and after a two-day course among 20 orthopedic trauma surgeons in an Arbeitsgemeinschaft für Osteosynthesefragen (Swiss working group for bone fusion issues) advanced trauma course on complex elbow fractures [23]. The eponym questions covered the areas of surgical techniques, fracture types, injury, and pathologic findings. The training involved didactic and cadaveric sessions. In order to prevent bias, no emphasis was placed on the eponyms, and participants were unaware of the nature of the study. The study found that correct answers about the eponym improved in only one question with inter-rater reliability (Kappa score) of 0.31 and 0.37 before and after the course, respectively [23]. Findings from this limited study suggest that this is an ineffective method for teaching eponyms.

We believe that eponyms are interesting to learn, and information about them should be retained and taught using an evidence-based approach. When teaching eponyms, we recommend that the components include a brief historical perspective of the person who described the sign, its original description, and its application in clinical practice. The teacher should explain and, in some cases, demonstrate the proper performance of the eponym and its application in clinical practice. The eponym should be practiced with feedback provided, and in the case of eponyms involving signs, their utility in assisting in diagnosis be emphasized [5,24].

\section{Conclusions}

Teaching and learning eponyms in the context of a historical perspective and, as described by the author, tells a story using a case-based learning applied model approach. This way of learning provides a meaningful way best to understand the application of eponyms in clinical practice. There is a continued impetus to remove eponyms and to substitute them using more descriptive terms. Although we do not favor this approach, we emphasize that eponyms must first be studied before devoting time to this endeavor to determine their effectiveness in clinical medicine. Those eponyms deemed to have utility in clinical practice should be retained and classified using predetermined criteria.

\section{Additional Information}

Disclosures 
Human subjects: All authors have confirmed that this study did not involve human participants or tissue. Animal subjects: All authors have confirmed that this study did not involve animal subjects or tissue. Conflicts of interest: In compliance with the ICMJE uniform disclosure form, all authors declare the following: Payment/services info: All authors have declared that no financial support was received from any organization for the submitted work. Financial relationships: All authors have declared that they have no financial relationships at present or within the previous three years with any organizations that might have an interest in the submitted work. Other relationships: All authors have declared that there are no other relationships or activities that could appear to have influenced the submitted work.

\section{References}

1. Woywodt A, Matteson E: Should eponyms be abandoned? Yes. BMJ. 2007, 335:424. 10.1136/bmj.39308.342639.AD

2. Whitworth JA: Should eponyms be abandoned? No. BMJ. 2007, 335:425. 10.1136/bmj.39308.380567.AD

3. Cogan DG: The rise and fall of eponyms . Arch Ophthalmol. 1978, 96:2202-3. 10.1001/archopht.1978.03910060504003

4. Babu AN, Kymes SM, Carpenter Fryer SM: Eponyms and the diagnosis of aortic regurgitation: what says the evidence?. Ann Intern Med. 2003, 138:736-42. 10.7326/0003-4819-138-9-200305060-00010

5. Yale SH, Tekiner H, Mazza JJ, Yale ES, Yale RC: Cardiovascular eponymic signs: diagnostic skills applied during the physical examination. Springer International Publishing, Cham; 2021.

6. Bartolucci S, Forbis P: Stedman's medical eponyms. Lippincott Williams \& Wilkins, Baltimore; 2005.

7. Birrer RB, Birrer CD: Medical diagnostic signs: a reference collection of eponymic bedside signs . Charles C. Thomas, Springfield; 1982.

8. Dobson J: Anatomical eponyms. E \& S Livingstone Ltd, London; 1962.

9. Gould GM: Table of eponymic diseases . An illustrated dictionary of medicine, biology and allied sciences. Kiston, Son \& Co, Philadelphia; 1894. 380-92.

10. Havard C: Medical eponyms: diseases, syndromes and signs. Barry Rose Law Publishers Ltd, Chichester, West Sussex; 1998.

11. Jablonski S: Jablonski's dictionary of syndromes \& eponymic diseases . Krieger Publishing Company, Florida; 1991.

12. Thornton SP: Ophthalmic eponyms: an encyclopedia of named signs, syndromes, and diseases in ophthalmology. Aesculapius Publishing Company, Birmingham, Alabama; 1967.

13. Whonamedit? A dictionary of medical eponyms . Accessed: August 10, 2021: https://www.whonamedit.com/.

14. Rolleston H: Diseases described by medical men who suffered from them . Lancet. 1921, 197:836-8. 10.1016/S0140-6736(01)25036-3

15. Persaud TVN: Classification and nomenclature of morphological defects. Lancet. 1975, 1:513.

16. World Health Organization best practices for the naming of new human infectious diseases . (2015). Accessed: August 25, 2021: https://apps.who.int/iris/bitstream/handle/10665/163636/WHO_HSE_FOS_15.1_eng.pdf? sequence $=1$ \&isAllowed $=\mathrm{y}$.

17. Hennekam RC, Biesecker LG, Allanson JE, Hall JG, Opitz JM, Temple IK, Carey JC: Elements of morphology: general terms for congenital anomalies. Am J Med Genet A. 2013, 161A:2726-33. 10.1002/ajmg.a.36249

18. Gordon-Taylor G: In defense of eponyms. J Roy Coll Surg Edinb. 1959, 4:105-20.

19. Ersöz MG, Hocaoğlu M, Sayman Muslubaş IB, Arf S, Karaçorlu M: Vitrectomy due to vitreous hemorrhage and tractional retinal detachment secondary to Eales' disease. Turk J Ophthalmol. 2021, 51:102-6. 10.4274/tjo.galenos.2020.43709

20. Gittinger JW Jr: On eponyms. Surv Ophthalmol. 2021, 66:411. 10.1016/j.survophthal.2021.01.004

21. Margo CE: Bell's palsy and the peril of eponyms . Ophthalmic Plast Reconstr Surg. 2021, 10.1097/IOP.0000000000002048

22. Adie WJ: Complete and incomplete forms of the benign disorder characterized by tonic pupils and absent tendon reflexes. Br J Ophthalmol. 1932, 16:449-61. 10.1136/bjo.16.8.449

23. Viveen J, Somford MP, Koenraadt KLM, van den Bekerom MPJ, Eygendaal D, Schipper IB, Doornberg JN: The use of eponyms for surgical approaches and fractures in elbow surgery: accuracy and reliability pre- and post-training. Arch Bone Jt Surg. 2019, 7:191-8.

24. Garibaldi BT, Zaman J, Artandi MK, Elder AT, Russell SW: Reinvigorating the clinical examination for the 21st century. Pol Arch Intern Med. 2019, 129:907-12. 10.20452/pamw.15073 